\title{
Influência da Maturação Sexual no Limiar de Lactato em Jogadoras de Futebol
}

\author{
Influence of Sexual Maturation \\ in Lactate Threshold in Female Soccer Players
}

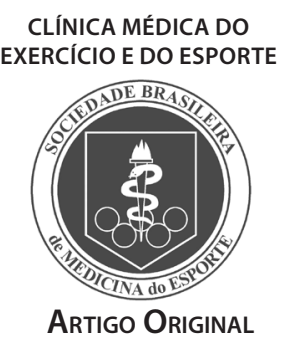

\author{
Ana Lucia Anauate Nicolao ${ }^{1}$ \\ André Pedrinelli ${ }^{1}$ \\ Paulo Sérgio Martino Zogaib' \\ Rogério Orbetelli' \\ Turíbio Leite de Barros Neto ${ }^{1}$ \\ 1. Unifesp - Universidade Federal \\ de São Paulo, Cemafe - Centro \\ de Estudos em Medicina da \\ Atividade Física e do Esporte e \\ Departamento de Cardiologia
}

Endereço para correspondência:

Av. Marechal Estenio de

Albuquerque Lima, no 82, porta 5

04005-040 - Paraíso - São Paulo, SP

E-mail: al.nicolau@uol.com.br

\begin{abstract}
RESUMO
Os limiares de lactato são utilizados para a avaliação da capacidade aeróbia em diferentes idades. Estudos demonstram que crianças e adolescentes apresentam menores concentrações sanguíneas de lactato [La], para mesma carga de esforço, do que adultos. Existem evidências de que isto está relacionado ao desenvolvimento maturacional das mesmas. Objetivo: Verificar a associação entre a maturação sexual e o limiar de lactato de atletas de futebol de 12 a 15 anos de idade. Método: A amostra foi do tipo intencional, não probabilística, com um total de 36 meninas, entre 12 a 15 anos, participantes de escolinhas de futebol da prefeitura da cidade de São Paulo. Foram obtidas da amostra a massa corporal, a estatura e a tomada de dobras cutâneas triciptal e panturrilha. A maturação sexual foi feita através da observação direta, por uma médica, do desenvolvimento de órgãos genitais e de pilosidade púbica, por meio de planilhas propostas por Tanner. Para determinação do limiar de lactato foi realizado teste progressivo em pista, onde as jovens realizaram três corridas de 800 metros, com a intensidade do esforço sendo controlada por zonas de frequência cardíaca pré-estabelecidas, com mensurações das [La] no final de cada corrida. Através da interpolação linear foi encontrada a velocidade correspondente a [La] de 2,5 mmol $\left(\mathrm{V}_{2,5}\right)$. Para compreender melhor a natureza das associações entre as variáveis foi utilizada a regressão linear múltipla, tendo como variável dependente o limiar de lactato $\left(\mathrm{V}_{2,5}\right)$, e como variáveis independentes idade (anos), IMC ( $\left.\mathrm{kg} / \mathrm{m}^{2}\right)$, estatura $(\mathrm{cm})$ e somatório de dobras cutâneas $(\mathrm{mm})$. Resultado: Em mais jovens, pré-púbere, as variáveis de crescimento e a maturação sexual tem pouca associação com o limiar de lactato. Sendo importante uma ponderação sobre a influência da maturação sexual no limiar de lactato. Conclusão: Levando em consideração a homogeneidade do grupo e o fato de a idade entre 12 e 15 anos ser um período de diversas modificações, o desenvolvimento maturacional, e não a idade cronológica, mostrou uma diferença significante nas variáveis analisadas.
\end{abstract}

Palavras-chave: capacidade aeróbia, concentração de lactato, futebol feminino.

\begin{abstract}
The lactate thresholds are used to assess the aerobic capacity in different ages. Studies show that children and adolescents present less lactate blood concentrations [La] than adults under certain effort loads. There are evidences that this is related to their maturational development. Objective:To verify the association between the sexual maturation and the lactate threshold in some adolescent soccer players ranging from 12 to 15 years old. Method: The sample was related to the intentional and not probabilistic type involving 36 girls, from 12 to 15 years old, members of the soccer schools held by the Mayority of São Paulo. The body weight, height and the sum of two skinfolds - calf and triceps were obtained from the sample. A physician directly observed the sexual maturation of the genitals and pubic hair development through the Tanner index.To determine the lactate threshold a progressive test, a 3x800m in running track, was performed by adolescents, their effort intensity was controlled by pre-established heart rate zones and the lactate blood concentrations [La] were measured at the end of each run. The velocity corresponding to lactate blood concentrations [La] of $2,5 \mathrm{mmol}(\mathrm{V} 2,5)$ was obtained through the linear interpolation.The multiple linear regression was used to better understand the nature of these associations between the variables, considering the lactate threshold (V2,5) as a dependent variable and the age (years), the body weight index $(\mathrm{kg} / \mathrm{m} 2)$, height $(\mathrm{cm})$ and the sum of the skinfolds $(\mathrm{mm})$ as independent variables. Result: The growth variables and the sexual maturation have little association with the lactate threshold in the youngest and pre-adolescent girls. It is important to take in consideration the sexual maturation influence on the threshold lactate. Conclusion: Taking in account the group homogeneity and being the range between 12 and 15 years old a period susceptible to many modifications, the maturational development and not the chronological age showed to be reponsible for a significative difference in the analysed variables.
\end{abstract}

Keywords: aerobic capacity Lactate concentration. Soccer Adolescents.

\section{INTRODUÇÃO}

A estrutura do futebol feminino no Brasil ainda é muito precária, mas nem por este motivo, podemos deixar de utilizar a avaliação funcional como método para verificar como está a qualidade física de nossos atletas. O controle fisiológico das cargas de treinamento provenientes da avaliação funcional é uma medida que deve ser valorizada, pois as informações obtidas podem determinar treinamentos objetivos para a melhora da aptidão física da atleta.

Existe uma procura permanente de melhores métodos de treinamento e de meios confiáveis de avaliar a aptidão física aeróbia dos atletas. Durante muito tempo, o volume máximo de absorção, transporte e 
utilização de oxigênio em unidade de tempo ( $\mathrm{VO}_{2}$ máx), foi considerado o fator biológico discriminador da aptidão física de esportistas praticantes de atividades com alta demanda energética aeróbia. Porém, a partir dos anos 70, constatou-se que o $\mathrm{VO}_{2}$ máx, isoladamente, não permite explicar o desempenho atlético nestas atividades, pois foram encontradas diversas combinações entre esse indicador e rendimento esportivo ${ }^{(1)}$.

Como abordagem padrão de variáveis submáximas, a medida de concentração sanguínea de lactato [la] é parte da rotina de vários laboratórios de fisiologia do exercício e avaliação funcional. Os chamados limiares de transição que, basicamente, refletem pontos onde ocorrem aumentos abruptos na curva [la]-intensidade, passaram a ser utilizados como referência de capacidade aeróbia (2). Em verdade, os limiares são aproximações da zona de intensidade no exercício no qual ocorreria um equilíbrio entre a produção e a remoção do lactato no sangue, a intensidade de máximo steady-state de lactato (MEEL). A determinação de limiares, principalmente o limiar de lactato (LL), em alguns trabalhos, denominado de limiar anaeróbio (LAn), é utilizada como referência de intensidade para a prescrição das cargas de capacidade aeróbia(3)

A [la] de $4.0 \mathrm{mmol}^{-L^{-1}}$ é frequentemente utilizada como indicadora do LAn e MEEL em adultos, sendo que muitas crianças podem suportar cargas próximo à exaustão sem exceder este valor de [la] ${ }^{(4)}$, tornando discutível a sua utilização como critério para avaliar os mais novos. Assim, foram sugeridos o uso de critérios com menores valores de CFL, como 2.5 mmol.L-1(5,6,7).

\section{Objetivo Geral}

Verificar a associação entre a maturação sexual e o limiar de lactato de jovens do sexo feminino de 12 a 15 anos de idade.

\section{Objetivos Específicos}

Verificar as relações existentes entre o limiar de lactato com outros indicadores de crescimento e desenvolvimento, como a idade cronológica, estatura, índice de massa corporal e somatório de dobras cutâneas (triciptal e panturrilha).

\section{MÉTODO}

\section{Amostra}

A coleta foi realizada após o consentimento dos pais, com aprovação do comitê de ética e pesquisa da Universidade Federal de São Paulo.

A amostra foi de 36 meninas entre 12 e 15 anos, jogadoras de futebol da prefeitura de São Paulo.

Para avaliar a fase da adolescência, transformou-se a idade cronológica em fase de maturação sexual. Deste modo, atletas de 12 anos foram considerados pré-púberes, 13 - 14 anos púberes, e as atletas de 15 anos foram consideradas pós-púberes.

\section{Medidas Antropométricas}

Foram obtidas da amostra a massa corporal, a estatura, e as dobras cutâneas triciptal e panturrilha, para efetuar a somatória das dobras cutâneas ( $\mathrm{DCC})$ (tabela 1).

O peso foi mensurado com uma balança digital portátil filizola com precisão de $100 \mathrm{gr}$. A estatura foi mensurada com estadiômetros de metal (precisão de $1 \mathrm{~mm}$ ), com a criança em posição ortostática, com o peso do corpo distribuído em ambas as pernas. As dobras cutâneas foram mensuradas com um adipômetro Cescorf, com precisão de medida de $0.1 \mathrm{~mm}$. As medidas foram realizadas de acordo com a padronização de Slaughter ${ }^{(8)}$.

Para determinação do limiar de lactato foi realizado teste progressivo em pista, (tabela 2, gráfico 1) em analogia ao estudo de Frainer et $a l^{(9)}$
Tabela 1. Características antropométricas da amostra.

\begin{tabular}{c|c|c|c|c|c|c|c|c}
\hline & \multicolumn{2}{|c|}{ Geral } & \multicolumn{2}{c|}{ Pré-púbere } & \multicolumn{2}{c|}{ Púbere } & \multicolumn{2}{c}{ Pós-púbere } \\
\cline { 2 - 9 } & Média & DP & Média & DP & Média & DP & Média & DP \\
\hline Idade & 13,17 & 1,16 & 11,92 & 0,29 & 13,00 & 0,00 & 14,58 & 0,51 \\
\hline Peso $(\mathrm{Kg})$ & 49,33 & 7,47 & 43,63 & 6,03 & 51,33 & 6,77 & 53,04 & 6,37 \\
\hline Estatura $(\mathrm{cm})$ & 155,67 & 5,01 & 151,25 & 5,35 & 156,67 & 2,84 & 159,08 & 2,84 \\
\hline IMC & 20,29 & 2,55 & 19,00 & 2,03 & 20,90 & 2,65 & 20,97 & 2,61 \\
\hline Soma DC & 36,00 & 7,76 & 32,33 & 7,98 & 39,92 & 6,64 & 35,75 & 7,24 \\
\hline \% Gordura & 27,06 & 4,73 & 24,82 & 4,87 & 29,45 & 4,05 & 26,91 & 4,42 \\
\hline
\end{tabular}

As atletas realizaram três corridas de 800 metros, com a intensidade do esforço controlada através da zona de frequência cardíaca pré-estabelecida 75\%, 80\% e 85\% da frequência cardíaca máxima,

Tabela 2. Descritivo das medidas de lactato, tempo dos tiros e velocidades dos limiares.

\begin{tabular}{c|c|c|c|c|c|c|c|c}
\hline & \multicolumn{2}{|c|}{ Geral } & \multicolumn{2}{c|}{ Pré-púbere } & \multicolumn{2}{c|}{ Púbere } & \multicolumn{2}{c}{ Pós-púbere } \\
\cline { 2 - 9 } & Média & DP & Média & DP & Média & DP & Média & DP \\
\hline LAC REP & 1,53 & 0,24 & 1,43 & 0,21 & 1,61 & 0,24 & 1,57 & 0,25 \\
\hline LAC 1 & 2,47 & 0,49 & 2,19 & 0,27 & 2,60 & 0,41 & 2,61 & 0,64 \\
\hline LAC 2 & 2,99 & 0,70 & 2,51 & 0,40 & 3,22 & 0,68 & 3,24 & 0,76 \\
\hline LAC 3 & 3,83 & 1,08 & 3,04 & 0,74 & 4,14 & 0,99 & 4,32 & 1,05 \\
\hline Tempo 1 (s) & 246,28 & 8,20 & 248,50 & 5,70 & 248,50 & 9,09 & 241,83 & 8,17 \\
\hline Tempo 2 (s) & 240,44 & 8,01 & 240,50 & 5,93 & 244,08 & 6,36 & 236,75 & 9,96 \\
\hline Tempo 3 (s) & 238,86 & 12,07 & 238,25 & 7,03 & 239,58 & 7,89 & 238,75 & 18,74 \\
\hline Vel lim (m/s) & 2,87 & 0,65 & 3,18 & 0,28 & 2,77 & 0,67 & 2,74 & 0,79 \\
\hline Vel lim (km/h) & 10,34 & 2,35 & 11,45 & 1,02 & 9,97 & 2,41 & 9,88 & 2,84 \\
\hline
\end{tabular}

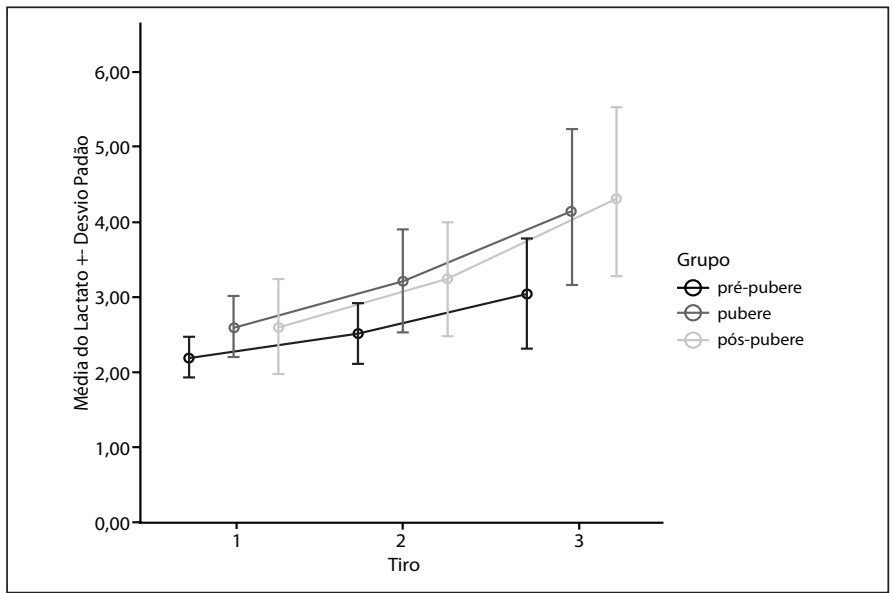

Gráfico 1. Média de lactato por tiro

respectivamente para cada corrida, metodologia modificada por Geysemeyer e Rieckert ${ }^{(10)}$.

Os procedimentos do teste foram:

O teste foi realizado em pista de atletismo;

Aquecimento de 10 minutos com corrida contínua de baixa intensidade e 10 minutos de exercícios de alongamento. Cinco minutos após foi realizada a primeira corrida de $800 \mathrm{~m}$, em que a atleta manteve a FC entre 140-150bpm; as outras corridas foram realizadas em valores de FC entre 160-170bpm e 180-190bpm, controlados a partir do monitor de frequência cardíaca, marca polar, com registro do tempo resultante de cada corrida de $800 \mathrm{~m}$.

A coleta de sangue para mensuração das [la] foi realizada imediatamente após cada corrida de $800 \mathrm{~m}$. O intervalo entre as mesmas foi de 1 minuto.

Para a medida da FC foi utilizado monitores de FC Polar (Polar Electro ${ }^{\circledR}$ ). As medidas das [la] no sangue foram analisadas pelo método eletroenzimático, em um aparelho yellow springs instruments, com amos- 
tras de 25 microlitros de sangue capilar retirado do dedo indicador.

A variável determinada no teste foi a velocidade media na concentração de lactato de 2,5mmol.

Foi avaliada a maturação sexual por meio de planilhas de avaliação propostas por Tanner ${ }^{(11)}$ e já validadas para crianças brasileiras por Matsudo e Matsudo ${ }^{(12)}$.

A escala de Tanner é uma escala de desenvolvimento físico em crianças e adolescentes. A escala define medidas físicas de desenvolvimento, baseado nas características sexuais secundárias, tais como o tamanho dos seios, genitália e desenvolvimento de pêlos pubianos (tabela 3). Dividida em cinco estágios:

Tabela 3. Descritivo da maturação sexual.

\begin{tabular}{c|c|c|c|c|c|c|c|c}
\hline Maturação & \multicolumn{2}{|c|}{ Geral } & \multicolumn{2}{c|}{ Pré-púbere } & \multicolumn{2}{c|}{ Púbere } & \multicolumn{2}{c}{ Pós-púbere } \\
\hline & \multicolumn{2}{|c|}{$\mathbf{N}=\mathbf{3 6}$} & \multicolumn{2}{|c|}{$\mathbf{N}=\mathbf{1 2}$} & \multicolumn{2}{c|}{$\mathbf{N}=\mathbf{1 2}$} & \multicolumn{2}{c}{$\mathbf{N}=12$} \\
\cline { 2 - 10 } & $\mathbf{N}$ & $\%$ & $\mathbf{N}$ & $\%$ & $\mathbf{N}$ & $\%$ & $\mathbf{N}$ & $\%$ \\
\hline M2-P2 & 7 & 19,44 & 6 & 50,00 & 1 & 8,33 & 0 & 0,00 \\
\hline M2-P3 & 9 & 25,00 & 3 & 25,00 & 2 & 16,67 & 4 & 33,33 \\
\hline M3-P3 & 7 & 19,44 & 3 & 25,00 & 4 & 33,33 & 0 & 0,00 \\
\hline M4-P3 & 1 & 2,78 & 0 & 0,00 & 1 & 8,33 & 0 & 0,00 \\
\hline M4-P4 & 9 & 25,00 & 0 & 0,00 & 4 & 33,33 & 5 & 41,67 \\
\hline M4-P5 & 1 & 2,78 & 0 & 0,00 & 0 & 0,00 & 1 & 8,33 \\
\hline M5-P5 & 2 & 5,56 & 0 & 0,00 & 0 & 0,00 & 2 & 16,67 \\
\hline
\end{tabular}

Tanner I Sem pêlos pubianos (pré-púberes).

Tanner II Pequena quantidade de cabelo longo, míldio com pequena pigmentação nos grandes lábios.

Tanner III O cabelo se torna mais grosso e crespo, e começa a se estender lateralmente.

Tanner IV A qualidade do cabelo, estendendo-se através.

Tanner $V \quad$ Cabelo se estende à superfície medial da coxa.

mamas

Tanner I Ausência de tecido glandular, aréola segue os contornos da pele do peito.

Tanner II Pequena área circundante tecido glandular, aréola começa a se alargar.

Tanner III Mama começa a tornar-se mais elevada, e se estende além das aréolas.

Tanner IV Aumenta o tamanho da mama, presença de papila..

Tanner $V \quad$ Mama atinge o tamanho adulto final.

A avaliação da maturação sexual foi feita através da observação direta, por uma medica, observando o desenvolvimento de órgãos genitais e de pilosidade púbica.

\section{Analise estatística}

As jovens foram dividas em três grupos pré-púbere, púbere e póspúbere. Para verificar a associação entre duas variáveis foi aplicada a correlação simples de Pearson.

Para verificar a associação entre a maturação sexual e as outras variáveis do estudo, foi aplicada a correlação de Spearman-rank.

O comparativo da estatura, peso e da somatória das dobras cutâneas nas idades cronológicas foi feita através do teste ANOVA.

Para todas as análises foi utilizado o nível de significância para $p<0,05$.

\section{RESULTADOS}

Comparando a idade cronológica corrigida, massa corporal, estatura e somatório de dobras cutâneas ( $\mathrm{DC}$ ) para a amostra das meninas pré-púbere, púbere e pós-púbere, utilizando como critério de púbere o início da menarca não foram encontradas diferenças significantes em nenhuma variável analisada (tabela 4, gráfico 2).

Tabela 4. Correlação de Spearman's entre maturação sexual e lactato sanguíneo nos tiros 1,2 e 3 .

\begin{tabular}{c|c|c|c|c|c}
\hline Grupos & Variável & & LAC 1 & LAC 2 & LAC 3 \\
\hline Pré-púbere & MAT M & Coef. Correlação & 0,2802 & 0,6761 & 0,6702 \\
\hline & & P-valor & 0,3777 & $0,0158^{*}$ & $0,0171^{*}$ \\
\hline & MAT P & Coef. Correlação & 0,2912 & 0,4392 & 0,8706 \\
\hline & & P-valor & 0,3584 & 0,1532 & $0,0002^{* *}$ \\
\hline Púbere & MAT M & Coef. Correlação & 0,5403 & 0,8242 & 0,8376 \\
\hline & & P-valor & 0,0697 & $0,0010^{* *}$ & $0,0007^{* *}$ \\
\hline & MAT P & Coef. Correlação & 0,5580 & 0,6521 & 0,7333 \\
\hline & & P-valor & 0,0594 & $0,0216^{*}$ & $0,0067^{* *}$ \\
\hline Pós-púbere & MAT M & Coef. Correlação & 0,7571 & 0,7507 & 0,8580 \\
\hline & & P-valor & $0,0044^{* *}$ & $0,0049^{* *}$ & $0,0004^{* *}$ \\
\hline & MAT P & Coef. Correlação & 0,7426 & 0,7177 & 0,7476 \\
\hline & & P-valor & $0,0057^{* *}$ & $0,0086^{* *}$ & $0,0052^{* *}$ \\
\hline
\end{tabular}

**Correlação é significante a $1 \%$.

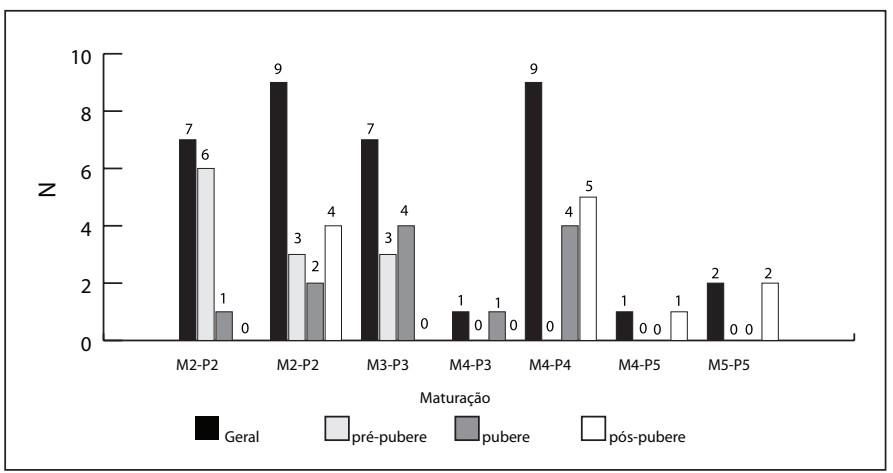

Gráfico 2. Maturação sexual

Comparando entre os grupos pré-púberes e pós-púberes foram encontradas diferenças significantes entre massa e estatura.

Comparando as variáveis de performance V2,5 (3,18 \pm 0,28; 2,77 \pm 0,67; 2,74 0 0,79m.s-1) e nas idades de pré-púbere, púbere e pós-púbere, respectivamente, não houve diferença significante entre os grupos.

Em mais jovens, pré-púbere, as variáveis de crescimento e a maturação sexual tem pouca associação com o limiar de lactato. Sendo importante uma ponderação sobre a influência da maturação sexual no limiar de lactato.

\section{DISCUSSÃO}

Quando o objetivo é direcionar e compreender melhor o que pode interferir ou auxiliar no desempenho humano, principalmente para que não se cometam erros pedagógicos que possam afetar a integridade física de crianças e adolescentes ao submetê-las ao treinamento e atividades que exijam de sobremaneira o seu organismo, faz-se necessária a relação entre os determinantes do crescimento e desenvolvimento e a capacidade de realizar esforços das mais variadas magnitudes ${ }^{(9)}$.

Nos resultados da maturação sexual, IMC, avaliados nas jogadoras de futebol feminino participantes deste estudo, foram encontradas diferenças significante na massa corporal e na $\sum D C$ entre os grupos. Também, podemos observar que houve uma grande variabilidade nos resultados dos índices de crescimento nesses grupos. Provavelmente, segundo Fagundes e Krebs, à associação que existe entre essas variáveis e o período de estirão de crescimento, onde nas meninas ocorre mais cedo em relação ao sexo masculino, por volta dos 12 anos. 
Quando as condições ambientais são ótimas, o genótipo é o primeiro regulador do crescimento e maturação. Entretanto, o ambiente social pode influenciar direta ou indiretamente através de fatores como nutrição, as relações familiares, tamanho da família, hábitos de atividade física, hábitos esportivos na família, na escola e na comunidade. Malina ainda conclui que a atividade física, por si só, não determina o crescimento e a maturação.

As variáveis de desempenho, $\mathrm{V}_{2,5}$, foram diferentes estatisticamente nos grupos, pré-púbere, púbere e pós-púbere, o que denota uma associação entre essas variáveis de desempenho da maturação sexual.

Não é possível fazer comparações entre os resultados deste estudo com outros estudos que tiveram objetivos semelhantes. Isso se deve, principalmente, às diferenças metodológicas.

Na análise de regressão, os índices de crescimento e a maturação sexual explicaram o desempenho em $V_{2,5}$ as variáveis de crescimento e a maturação sexual têm associação com o limiar de lactato. Assim, é importante fazer uma reflexão sobre a influência da maturação sexual no limiar de lactato.

Diversos pesquisadores se preocupam em investigar as razões, a partir dos estudos de Ericksson et al. ${ }^{(13,14)}$, pelos quais os mais jovens apresentavam menores concentrações sanguíneas de lactato. Uma das razões para essa limitação estaria relacionada a uma menor capacidade glicolítica e que consequentemente estaria ligada à maturação sexual.

No presente estudo, foi encontrada associação significante entre índices de maturação sexual e limiar de lactato.

\section{LIMITAÇÕES DA PESQUISA}

a) A abordagem amostral do presente estudo foi não probabilística, o que é uma ameaça de validade externa dos resultados obtidos;

b) Não foi determinada a intensidade de MEEL;

c) foi feita somente uma medida de [la] em cada intensidade de exercício.

\section{CONCLUSÃO}

A maturação sexual e os índices de crescimento, estão associados com o desempenho na velocidade do limiar.

Concluindo que meninas pré-púberes têm maiores velocidades no limiar de lactato do que as jovens pós-púberes devido a maturação sexual.

Nos resultados deste estudo, levando em consideração a homogeneidade do grupo e o fato de a idade entre 12 e 15 anos ser um período de diversas modificações, o desenvolvimento maturacional e não a idade cronológica mostrou uma diferença significante nas variáveis analisadas.

\section{AGRADECIMENTOS}

Agradecemos a toda equipe do CEMAFE que colaboraram diretamente nesse estudo.

Agradecimento especial ao professor Dr. André Pedrinelli ortopedista e especialista em medicina do esporte do Hospital das Clínicas de SP.

Todos os autores declararam não haver qualquer potencial conflito de interesses referente a este artigo.

\section{REFERÊNCIAS}

1. Noakes TD. Maximal oxygen uptake: "Classical" versus Contemporary viewpoints. Med Sci Sports Exerc 1998;30:1381-98.

2. Heck H, Hess G, Mader A. Comparative study of different lactate threshold concepts. Dsc Z Sportmed 1985;36:19-25.

3. Oliveira FR, Gagliardi JFL, Kiss MAPDM. Proposta de referências para a prescrição de treinamento aeróbio e anaeróbio para corredores de média e longa duração. Rev Paul Educ Fís 1994;8:68-72.

4. Williams JR, Armstrong N, Kirby BJ. The influence of age and maturation on the 2.5 and $4.0 \mathrm{mmol}$ levels of blood lactate in girls. J Sports Sci 1990:8:80.

5. Williams JR, Armstrong N, Kirby BJ. The relationship between children's blood lactate responses to incremental and constant speed treadmill exercise. J Sports Sci 1990;8:298-9.

6. Williams JR, Armstrong N, Kirby BJ. The blood lactate response to exercise in 11 to 16 -year-old children with reference to cardiorespiratory variables, chronological age, sex and maturity. J Sports Sc 1990;8:297-8

7. Armstrong N, Wellsman J. Aerobic exercise: growth and maturation. In: Young people \& physical activity.
New York: Oxford Univ. Press, 1997

8. Slaughter MH, Lohman TG, Boileau RA, Horswill CA, Stillman RJ, Vanloan MD, et al. Skinfold equations for estimation of body fatness in children and youth. Hum Biol 1998;60:709-23.

9. Frainer DES, De-Oliveira FR, Cal Abad CC, Kiss MAPDM. Evidências de validade do T20 como aproximação do limiar anaeróbio em jovens jogadores de futebol. R. da Educação Física/UEM 2004;15:33-7.

10. Geysemeyer U, Rieckert H. Field-step tests for sports discipline-related diagnosis of endurance power Int J Sports Med 1987;8:132.

11. Tanner J M. Growth at adolescence. Oxford: Blackwell Scientific, 1962

12. Matsudo SMM, Matsudo UKR. Osteoporose e atividade física. Rev Bras Ci e Mov 1991;5:33-54.

13. Ericksson BO, Karlsson J, Saltin B. Muscle metabolites during exercise in pubertal boys. Acta Physiol Scand 1971;87:27-39.

14. Ericksson BO. Physical training, oxygen supply and muscle metabolism in 11-13 years old boys. Acta Physiol Scand 1972:38:1-48. 